\title{
REGULATION OF ARTIFICIAL INTELLIGENCE: ONE PERSPECTIVE FROM THE THEORY OF GAMES
}

\author{
OCTAVIO JOSÉ SALCEDO PARRA ${ }^{1,2}$, DAVID JOSÉ SILVERA VILLEGAS ${ }^{2}$ \\ \& ROBERTO MANUEL POVEDA CHAVES ${ }^{1}$ \\ ${ }^{1}$ Faculty of Engineering, Universidad Distrital Francisco José de Caldas, Bogotá, Colombia \\ ${ }^{2}$ Faculty of Engineering, Universidad Nacional de Colombia, Bogotá D.C., Colombia
}

\begin{abstract}
The following document presents the analysis of a recent debate framed around artificial intelligence. Thus, from the theory of games a scenario is presented in that the different points of view are put in perspective and seeks to resolve the debate from the application of the game theory.

The document at the beginning puts in perspective the reader indicating the context on which the debate takes place. Then he presents a game model and continues with the resolution of the same for at the end of the document show the analysis and conclusions of the same.

KEYWORD: Artificial Intelligence, Games Theory \& Regulation.
\end{abstract}

Received: Oct 13, 2020; Accepted: Nov 03, 2020; Pbublished: Nov 11, 2020; Paper Id.: IJMPERDOCT202050

\section{INTRODUCTION}

In the last few years, it is not strange to read news about technological advances that have a direct impact on the society, here the multiple computer systems have a place, the internet, robots, among many other tools that are created with the initial philosophy of empowering and helping in the development of society. So, beyond the innovation, it's worth asking yourself how these tools they begin to interact with people; well, in the last who have a direct interaction with the machines and in a world in which there is greater access to technology, results important to know how it interacts with society. Within the large group of technologies that exist the subject of Artificial intelligence (AI) is particular, because if you goes back to famous films such as I Robot (2004), Terminator (1984), A.I. Artificial Intelligence (2001), among others; the situation that a machine, robot or similar has own thought makes a reaction develop (fear in some), due to their capacity, reach and intentions that they can develop.

Recently there was a debate between the founder of Tesla: Elon Musk and the founder of Facebook: Mark Zuckerberg. The first mentioned that should start be proactive against the implementation of technologies such as AI, before it's too late to react to unfavorable events as a result of poor development of the technology. For its part, the founder of Facebook, rejected strongly Musk's statements arguing that He was being incendiary and that some type is not necessary of regulation.

About who is the reason it is quite difficult to take a decision, however, although there are websites like The Atlantic [1] that say that the reactions are more focused to the interests of each of the companies that they 
handle; the certain thing is that it is possible to pose a scenario in which the technology as AI, which can also be extended to autonomous vehicles, home automation, personal assistants, among others; will make part of the daily life of people and considering a regulation of this type of technology is fully rational.

\section{BACKGROUND}

\subsection{Artificial Intelligence}

In [2] they define artificial intelligence as science and the engineering of making smart machines, especially computer programs. It is related to the fact of using computers to understand human intelligence, but artificial intelligence does not have to be related to biologically observable methods. Defining intelligence as the ability to achieve goals in the world. This from different planes, such as people, animals, machines among others.

There are positions that consider that the development of artificial intelligence can generate problems in the future for society. For example, the philosopher John Searle says that the idea of a non-biological intelligent machine is inconsistent [2], proposing the argument of the Chinese courtroom [5] which in summary indicates that machines cannot think and that this ability is typical of a biological evolution.

The Turing test was a test proposed by Alan Turing in 1950, where he describes the conditions to consider an intelligent machine In the test, the person who performs it is capable to recognize who is a machine and who is the human.

In addition, the conversation between the parties is limited by a keyboard or some textual medium and thus ignore the skill of the machine to talk. If for some reason who the evaluation does not distinguish between the texts of both parties. I could say that the machine has passed the test.

It is clear that the test only allows to measure the similarity between answers from each of the parties. In addition, another discord born when analyzing that the test only works in one direction: yes the machine passes the test, it can be considered intelligent;

However, you can be smart without knowing much without being a human being, therefore: could he really be intelligent?

As a similar example, we have the case where different machines can play against humans and defeat the best players in the world. To quote a couple of examples: in 1997, the IBM Deep Blue computer managed to beat the best chess player of that era after the contest 12 chess games for 2 years. And on the other side, in a game of greater complexity than chess: the Go. A computer in 2014 managed to beat the best player of Korean Go, winning 4 out of 5 games; something that enhances the work done in artificial intelligence techniques, [4]. Years later, that technology evolved to what IBM Developed as Watson: a developed supercomputer by IBM, which combines artificial intelligence and software of analysis to achieve a machine of Questions-Answers with applications in health, education, finance, among others.

\subsection{Related Works}

In [7], Ríos W. explains that there are initiatives such as of the Knight Foundation, the Massachusetts Institute of Technology (MIT), Harvard University and other institutions, who have decided to create the Fund for Ethics and Government of Artificial Intelligence (Ethics and Governance of the Artificial Intelligence Fund, in English), and initiatives such as that is ahead since January 20, 2015, where the Committee of the European Parliament for Legal Affairs, constituted a committee that allows for the legal and legal questions that they involve robotics and artificial intelligence. 
Likewise in several countries such as the USA, Japan, China and South Korea, where the rising wave of technology has had a speed enormously, they have begun to create and consider standards that allow these topics to be regulated and to allow us to delve into other plans like the legal one where before it was not thought that could be said technologies.

On the other hand, in [3], NY Times puts in perspective two actors who recently clashed in a fight to defend his position against artificial intelligence. First is a tool with a high potential for harm, understood as a potential risk of being a misused tool and therefore, it can represent a risk for societies. In counterpart, the creator of Facebook Marck Zuckemberg said that Musk's statements were pretentious because he was being alarmist and creating a false idea of the ability and use of artificial intelligence. That's why Etzioni O. [3], proposes 3 rules that should regulate developments and advances from artificial intelligence. The first rule is that the machines, robots, etc; they must be subject to the regulations who relay as a human on their operator. The second is that systems that have AI must clearly differentiate what is and what is not a human and that his machine condition does not allows you to be human and you must maintain that position throughout of its use. Finally, the third rule indicates that the systems with AI they should not withhold information and everything that arrives to them (Sensors, big data, etc.) must be shared with whom the system operates; this to have a control over the information, its integrity and the operation of it. The European Commission sets out in its report [8] a series of considerations and suggestions for initiating legislation about robotics and machines that have intelligence artificial. Initially they propose a common definition of robot autonomous intelligent that includes the following features:

- It has a series of devices that allow you to take the information about your environment and interact with it (sensors).

- He has the ability to have autonomy in learning.

- There is a physical structure that supports it,

- Allows you to change your behavior as you perform it the environment.

It is also suggested the creation of a system that allows register robots and a system that establishes criteria for the classification of the robots, in order to be able to know those with the operation, processing capacity and enough technology to have to be registered.

In addition to the technical principles, he proposes a series of ethical principles that are born from the close relationship that it can occur between societies and robotics. Initially ratifies the position that justifies that robotics touches themes and sometimes puts at risk some issues such as security human, autonomy in data management, freedom, and privacy and among others [8].

In addition, he also believes that a legal framework is pertinent and ethic that serves as a north when making designs and implementation of the robots, and thus be able to give full to the legal recommendations postulated throughout the document. Finally, it proposes a framework that involves a code of conduct a code of morals and licenses for those who work with robotics, for ethics committees have the task of reviewing the protocols in robotics and for all kinds of designers and users. Finally, name another type of legislation that involves robotics in different areas: medicine, autonomous vehicles, international level, and among others.

In [6], Xiuquan Li and Tao Zhang, indicate that there are 3 areas where there may be problems with the machines and artificial intelligence. These three ambiguities are security, privacy and ethics, where it is argued that there are risks 
potential In the first area, the way in which security is vulnerable due to the abuse of technology, since the way AI is used

will change the impact that generate On the other hand, it is argued that technical defects do not they are foreign to a tool like this and therefore the errors They can cause problems for humans. Finally discusses a third risk due to the development and evolution of machines in what they call: strong artificial intelligence.

In privacy, it is argued that this can be compromised in the excessive use of sensors and information that we contribute to the network. Something that according to the authors, can generate clear privacy problems and compromise not only the information, but our own person.

Finally, he discusses the ethical problems and here he makes a strong analysis in the fact of regulating or not the robots that count with the ability to think due to AI. Also, what is the role of robots in a society human rights and if this role makes them creditors of a people.

In [7] Hurlburt G. discusses the reliability of intelligence and how much this reliability affects development. For this, he names the different methods with that the AI trains and shows different examples the way in which a tool like the AI can fail.

As arguments it indicates that broadly the intelligence artificial is software and that it is subject on the one hand to programmed skills (culture, social context, among others) and on the other hand is subject to failures since not always all the cases in which he will operate are known.

In addition, these arguments reinforces it with a series of examples that show that errors in these programs entail to lose human lives, as well as to cause damages to third parties within a community; the question is then "how trust the results of the AI "[7]. From that perspective it is possible to pose a position of rejection towards AI as tool of common use and wide coverage. And by On the other hand, it is possible to plan a more libertarian position than let's believe in a world with such a great tool reach and overcrowded as it is the AI.

\section{THE MODEL}

For the analysis and approach of the game, it will be assumed that game not cooperative between both parties, so then each one of the decisions of the players will not influence the decision of the other. In addition, given the form of play, it is possible an approach of matrix form from where it could be analyze each situation.

\subsection{To the Players}

Depending on the game you can consider two types of players:

- The precursors (P) of the opening of artificial intelligence to all common use objects for people, that is, those who are not in favor of regulation.

- The detractors (D) of a liberation of intelligence artificial as a tool for social and massive development, that is, who is in favor of a regulation.

\subsection{The Cases, Actions}

Two actions can be assumed for each of the actors, these actions are framed under assumptions of the behavior of each of the players and therefore, they may be subject to changes. However for the proposal of the game, the action is assumed to be simpler than according to discussions and texts consulted may be fully applicable. 
- P performs a campaign to massively extend the tool, incorporating massive strategies for its use. Delegitimizing and also contradicting the position from D.

- D carries out a regulation campaign in favor of the use of artificial intelligence and therefore its action is concentrated in showing the possible consequences of not regulating.

So then, according to these actions, a series can be assumed of payments that will be according to the profile of each one. There is $B_{D}$ payment that corresponds to the profit of $\mathrm{D}$, however in the If $\mathrm{D}$ manages to earn the benefit, he would have to make $\mathrm{C}$ payments that corresponds to the cost of implementing a regulation, and in addition to that, there would be a payment $t$ that would to influence the decision of the other player.

On the other hand $\mathrm{P}$, can earn a $\mathrm{B}_{\mathrm{P}}$ payment product of winning the debate. Although in case of winning the own benefit, you should also make an S payment that corresponds to the payment for carrying out campaigns to use the AI.

With this in mind, you can assume the following order of payments:

$$
B_{D}>B_{P}>t>C>S
$$

- Game \# 1: For the first game the following will be assumed possibilities, where the first possibility would be to greater interest for each player. In this game, they pose two positions: $\mathrm{P}$ will be in favor of D's actions; in case otherwise, it will be against D's regulatory actions.

With this in mind, it is possible to raise the preferences of $\mathrm{P}$.

- $\quad \mathrm{P}$ is in favor of the non-regulation made by $\mathrm{D}$, obtaining so the $\mathrm{B}_{\mathrm{P}}$ benefit, but incurring the payment $\mathrm{S}$, since it must generate the teaching conditions adequate of the AI.

- $\quad \mathrm{P}$ is against the regulation of $\mathrm{D}$ and product of this situation would have the benefit $\mathrm{t}$.

- In this case, even though he is against $\mathrm{P}$, he would prefer that $\mathrm{D}$ do not want to regulate. Here, both players would not have any payment since it is the strategy that least would happen and a priori it would be the least probable strategy.

- Finally the last case is where $\mathrm{P}$ is in favor of the regulation imposed by $\mathrm{D}$ and hence would have the worst payment.

In the opposite case, for D the following actions would be assumed: A strategy that seeks to regulate AI and a strategy it seeks not to regulate the AI.

- D seeks a regulation and P decides to be in favor. For In this case, there would be a BD payment product of winning the game, however, would have to incur payments for ensure that adequate regulation is achieved $\mathrm{C}$.

- D seeks regular and P happens to be against. Here D would appeal to influence the decision of P and would have to perform a payment $\mathrm{t}$.

- $\quad \mathrm{D}$ despite its no regulatory position, $\mathrm{P}$ would be in against it and the payment as in the previous case, would be 0 . 
- Finally the last case is given with $\mathrm{P}$ being in favor and D not wanting to regulate, since D would incur a payment of $-\mathrm{B}_{\mathrm{D}}$.

Under these rules, it is possible to obtain the following matrix of payments shown in Table 1.

Table 1: Matrix of Payments for the Game 1. Where the First Position $n$ of Each Couple of the Matrix Corresponds to the Payment of $P$ and the Second Position of Each Couple Corresponds to the Payment of D

\begin{tabular}{|l|l|l|l|}
\hline \multirow{2}{*}{ Game 1 } & \multicolumn{2}{c|}{$D$} \\
\cline { 3 - 4 } \multicolumn{2}{|c|}{} & Regular & No Regular \\
\hline \multirow{3}{*}{$P$} & In favor & $\left(-B_{P}, B_{D}-C\right)$ & $\left(B_{P}-S,-B_{D}\right)$ \\
\cline { 2 - 4 } & Against & $(t,-t)$ & $(0,0)$ \\
\hline
\end{tabular}

This game is possible to resemble a classic game of prisoner, since in the game two perspectives are put independent players where each player will seek to maximize gain at the expense of the other that is, it is a non-cooperative game.

In [9], a situation arises in which there is an entity regulator and a player looking for ways to influence the regulating player There it is explained that the interests of each player may be affected or benefited by the way to act of each player. It is then possible to assimilate the game previously described with a prisoner game.

If $\mathrm{P}$ chooses to be against, he will always prefer that $\mathrm{D}$ choose regular, as this could bring you a benefit at the expense of interest of D to want to influence and put the game in his favor; the which is opposed to the other condition in which if $\mathrm{D}$ does not regulate, he simply will not receive any benefit.

Now, if it choose to be in favor, always prefer that the D's position is not regular, as this allows you to obtain all the possible benefit although you must incur a payment for having to teach how to use AI. Of course, under the assumptions of equation 1, this payment would be much less than the profit you can get and clearly would be better than the opposite position in which you would have a negative payment.

On the other hand, if $\mathrm{D}$ takes a perspective of not regular always $\mathrm{I}$ would prefer that $\mathrm{P}$ is against it since no payments would be received (although it is clear that it would be the position with less probability of occurrence). On the other hand, if you choose regular, you will always prefer that $\mathrm{P}$ is in favor of it and thus receive the best payment can. However, given that it is an unlikely position for $\mathrm{P}$, the nature of the players would make the game deviate to the position where D can influence $\mathrm{P}$ and $\mathrm{P}$ can accept or not this influence, which in the last resemble the most real position today.

It is possible to perform an initial verification of the game using the Gambit software [10] and assuming a series of payments according to the one presented in 1 . Suppose that $\mathrm{B}_{\mathrm{D}}=5, \mathrm{~B}_{\mathrm{P}}=4, \mathrm{t}=3, \mathrm{C}=2$ and $\mathrm{S}=1$, with these conditions you can make a first game whose matrix will be:

$$
\left[\begin{array}{cc}
(-4,3) & (3,-5) \\
(3,-3) & (0,0)
\end{array}\right]
$$

Where, to analyze the software, you can get the following results:

- Probability of the event $1: 1: 3 / 11$

- $\quad$ Probability of the event 1:2: 8/11 
- $\quad$ Probability of the event $2: 1: 3 / 10$

- $\quad$ Probability of the event 2:2: 7/10

It would be worth asking what would happen if there is a change in equation 1 and it is decided to do this time $\mathrm{S}>\mathrm{C}$, with $\mathrm{S}=2$ and $\mathrm{C}=1$. For this case, the payment matrix would be left following way:

$$
\left[\begin{array}{cc}
(-4,3) & (2,-5) \\
(3,-3) & (0,0)
\end{array}\right]
$$

- $\quad$ Probability of the event $1: 1: 1 / 4$

- $\quad$ Probability of the event $1: 2: 3 / 4$

- $\quad$ Probability of the event 2:1: 2/9

- $\quad$ Probability of the event 2:2: 7/9

\section{DISCUSSION OF RESULTS}

By observing the games raised above, it is possible see that for the first proposed game, when looking for the balance of Nash through the software, it is indicated that the probability higher in the event where D decides not to regulate and his part $\mathrm{P}$ decides to be in favor. That is, in this scenario it is indicated that the best option is not regular and for its part yes make a strong diffusion and teaching of the AI.

However, by making the change in which the payment for the teaching of AI is greater than the payment for regulation, the software tells us that the greatest probability and greater gain for both remains the 1: 2 scenario. Is say, where $\mathrm{D}$ decides not to regulate and $\mathrm{P}$ is in favor of it.

Now, it would be worthwhile to study what the probability would be of the occurrence of any of the 4 events and see who they are subject each of the probabilities in order to achieve identify which parameters make the achievement easier of one and another possibility. This is proposed, since for the two previous cases were considered as payments that were not necessarily have to be payments in reality and that they could chance.

For this, a probability that corresponds to the probability of D regulating $(\mathrm{R})$ and there is a probability $(\mathrm{F})$ which corresponds to the fact that $\mathrm{P}$ is in favor. Likewise, you can define the opposite cases, which will be the probability that both events do not occur: $(1-\mathrm{R})$ and $(1-\mathrm{F})$, respectively.

Defined these variables it is possible to find what would be the total gain of each of the players, based on the payment matrix and the probability that each event happen.

$$
\begin{aligned}
& U_{P}=-B_{P}(R)(F)+\left(B_{P}-S\right)(1-R) F+t(R)(1-F) \\
& U_{D}=\left(B_{D}-C\right)(R)(F)-\left(B_{D)}\right)(1-R) F-t()(1-F)(R)
\end{aligned}
$$

If each of the equations is derived with respect to the event of interest of each player $\left(\partial U_{P} / \partial F\right)$ and $\left(\partial U_{D} / \partial R\right)$, it is possible to find out what is the probability of an event occurring $u$ other; that based on the characteristics of each player you could get to a game trend from a perspective more global Equations 6 and 7 describe what is the probability that each event will happen. 


$$
\begin{aligned}
& R=\frac{S-B_{P}}{S-t-2 B_{P}} \\
& F=\frac{t}{2 B_{D}-C+t}
\end{aligned}
$$

To analyze equations 6 and 7, it is possible to observe that both strongly depend on the profit of each of the parts and assuming the condition given by 1 allows to see that the probability that Player P is in favor is small.

This is explained because the denominator would always be positive and it is strongly correlated with the payment of $\mathrm{D}$ that is the biggest, also knowing that always $\mathrm{t}>\mathrm{C}$ and that $\mathrm{B}_{\mathrm{D}}>\mathrm{t}$, the quantity will always tend to be less than 1 .

On the other hand, when analyzing the probability of D look for regular, you have that this probability is strongly conditioned for the $\mathrm{B}_{\mathrm{P}}$ payment and when analyzing the payments (based on 1) would have always that the amount would tend to be close or greater that 1 ; so in theory there is a greater probability that finally $\mathrm{D}$ look for regular, even when $\mathrm{P}$ is not in favor.

\section{CONCLUSIONS}

- In the two games analyzed, when giving a specific payment for the dads variables, it was obtained that the best condition of solution and balance for the parties involved; is one in which that group of people in favor of detract from the use of AI opts not to regulate and those who are in favor of its use should be in favor.

- In such a subjective debate it is possible to increase the number of variables that come into consideration and therefore propose a game more similar to reality.

- Given that in the real world an influence is possible between the parties involved, it is possible to see that the factor $\mathrm{t}$ has great weight in deciding the probability that the Player $\mathrm{P}$ is in favor of one or another situation.

- On the other hand, the probability that player D decides regular is conditioned by the payment that represents teach the proper use of AI to society.

- The limitation of a non-cooperative game does not allow evaluate other scenarios that may be able to adapt better to the real debate and therefore it would be worthwhile to carry out an analysis from this perspective.

\section{REFERENCES}

1. The Atlantic. Why Zuckerberg and Musk Are Fighting About the Robot Future. https://www.theatlantic.com/technology/archive/2017/07/muskvs-zuck/535077/. Consulta: Septiembre de 2017.

2. McCarthy, J. WHAT IS ARTIFICIAL INTELLIGENCE?. http://wwwformal.stanford.edu/jmc/whatisai/. Consulta: Septiembre de 2017

3. How to Regulate Artificial Intelligence: https://www.nytimes.com/2017/09/01/opinion/artificialintelligenceregulations-rules.html

4. Google's AI Wins Fifth And Final Game Against Go Genius Lee Sedol: https://www.wired.com/2016/03/googlesai-wins-fifth-final-game-gogenius-lee-sedol/

5. John Searle's Chinese Room Argument: www-formal.stanford.edu/jmc/chinese.html

6. An Exploration on Artificial Intelligence Application: From Security, Privacy and Ethic Perspective 
7. La regulación legal de la robótica y la inteligencia artificial: https://www.ambitojuridico.com/bancoconocimiento/tic/la-regulacionlegal-de-la-robotica-y-la-inteligenciaartificial

8. Report: https://www.europarl.europa.eu/doceo/document/A-8-2017-0005_EN.html

9. Albino, D. Hu, A. Bar-Yam, Y. Corporations and Regulators:The Game of Influence in Regulatory Capture. Cambdrige. 2013

10. Gambit - Software Tools for Game Theory: http://www.gambit-project.org/ 
